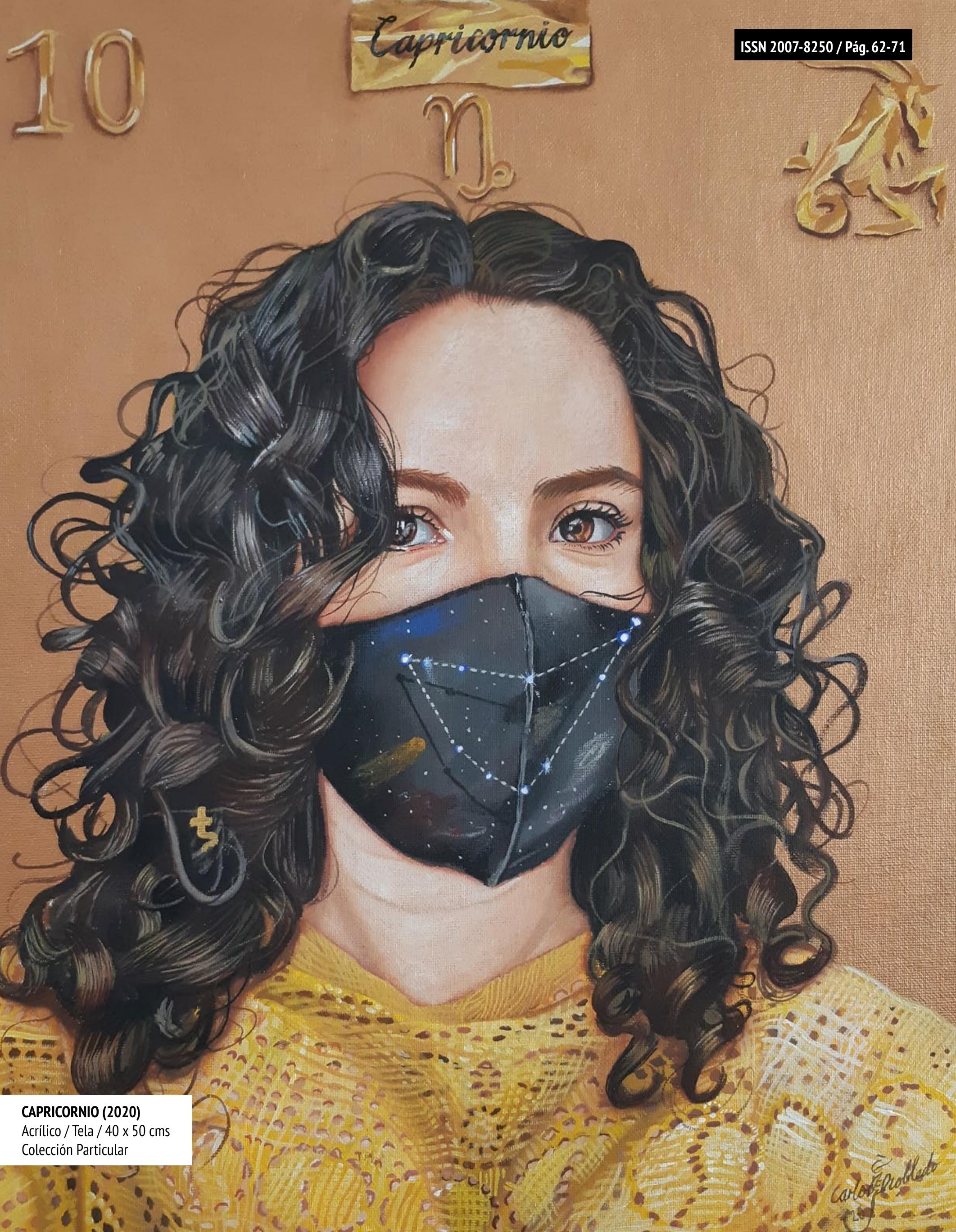




\section{https://doi.org/10.29105/pu8.16-6 \\ ORIENTACIÓN EDUCATIVA A DISTANCIA: EL VíNCULO ENTRE ESCUELA, ALUMNOS Y PADRES DE FAMILIA EN UN CONTEXTO DE DESIGUALDAD}

?

$$
\begin{aligned}
& \text { DISTANCE EDUCATIONAL GUIDANCE: THE LINK BETWEEN SCHOOL, } \\
& \text { STUDENTS AND PARENTS IN A CONTEXT OF INEQUALITY }
\end{aligned}
$$

\section{RESUMEN}

La educación a distancia por el confinamiento obligatorio a causa de la Pandemia COVID-19, fueron las indicaciones de las autoridades educativas en Educación Media Superior del Estado de México. Brindar atención a los estudiantes a través de la plataforma classroom, donde se subieron las actividades previamente por el docente y que debían realizar los estudiantes para el cumplimiento de los contenidos del plan de estudio. No obstante, las condiciones de educación a distancia, junto a clases virtuales a través de Meet y Zoom, se busca que exista interacción entre docentes y alumnos, atención a dudas e impartición de contenidos, con el propósito de diseñar una estrategia de acompañamiento y orientación para que los estudiantes tengan un desarrollo óptimo en la educación a distancia. En este documento se presentan los resultados obtenidos de la investigación que reflejan parámetros de la realidad de nuestra sociedad mexicana, ya que en algunas zonas y en ciertos aspectos, las desigualdades se hacen más presentes durante la Pandemia COVID-19 y de la misma manera lo que ocurre en las grandes ciudades 0 en zonas rurales, incluso al interior de una misma ciudad, dado que Ecatepec es parte de la zona metropolitana de la capital del país, y aun así existen este tipo de disparidades en las realidades que viven.

PALABRAS CLAVE:

Desigualdad, Oportunidad, Distancia, Disparidades, Educación, Orientación
ABSTRACT

Distance education due to mandatory confinement due to the COVID-19 Pandemic, were the indications of the educational authorities in $\mathrm{Hi}$ gher Secondary Education of the State of Mexico. Provide attention to students through the classroom platform, where the activities were previously uploaded by the teacher and that students had to carry out to comply with the contents of the study plan, despite the conditions of distance education, together with classes virtual through Meet and Zoom, it is sought that there is interaction between teachers and students, attention to questions and content delivery. With the purpose of designing an accompaniment and guidance strategy so that students have an optimal development in distance education. The results obtained reflect parameters of the reality of our Mexican society, since in some areas and in certain aspects inequalities become more present during the COVID-19 Pandemic and in the same way what happens in large cities or rural areas, even within the same city, since Ecatepec is part of the metropolitan area of the capital of the country, and even so there are such disparities in the realities they lives.

KEYWORDS: Inequality, Opportunity, Distance, Disparities, Education, Orientation 
D ebido a la pandemia Covid-19 el gobierno de México tomó la las clases presenciales y establecer un sistema de educación a distancia electrónica y digital, para la recuperación de contenidos de aprendizaje (SEP, 2020). En este sistema, los centros educativos diseñaron estrategias para impartir contenidos a distancia por internet y proteger el derecho a la educación de los estudiantes en nuestro país. De acuerdo con la Encuesta Nacional sobre Disponibilidad y Uso de Tecnologías de la Información en los Hogares (ENDUTIH) 2019, el 76.6\% de la población urbana es usuaria de internet, pero en zonas rurales la población usuaria es de $47.7 \%$, que en los hogares del país el $56.4 \%$ disponen de internet en una conexión fija o móvil y el $44.3 \%$ dispone de una computadora (INEGI, 2020).

Es necesario diseñar estrategias de acompañamiento y orientación para guiar a los estudiantes en la educación a distancia por la pandemia Covid-19, con la intención de que permanezcan en la escuela y que puedan abstraer los conocimientos necesarios para su formación y el tránsito hacia determinación de interrumpir

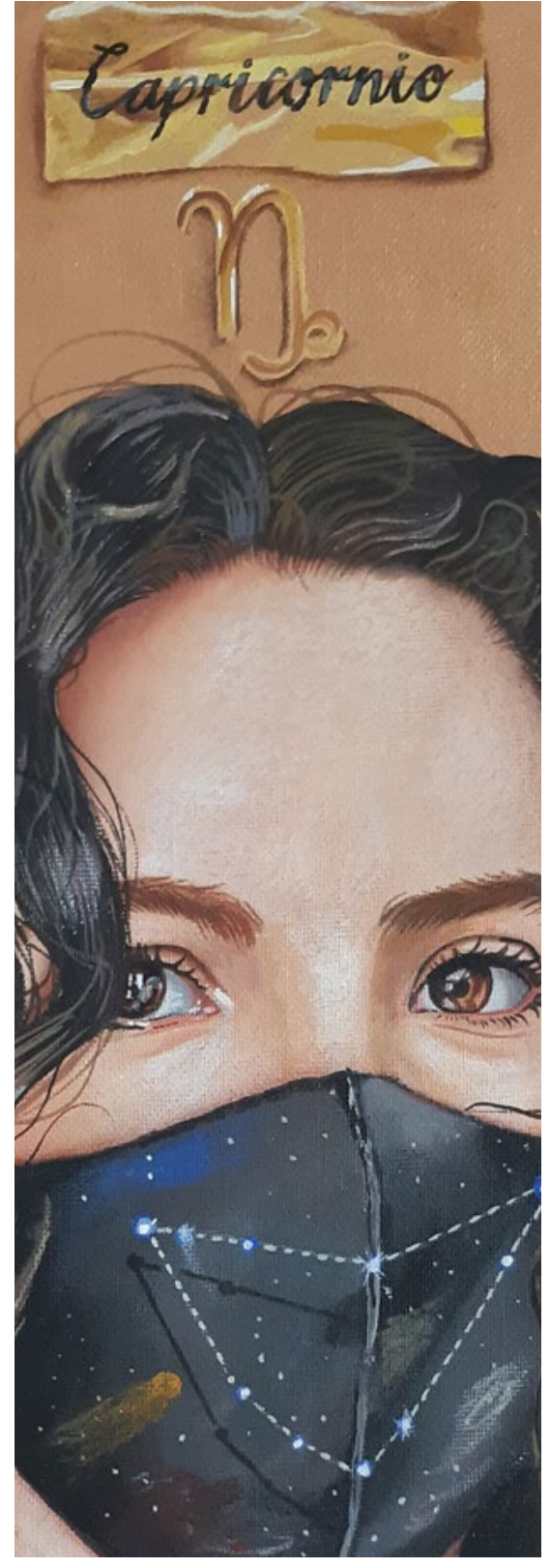

\section{Debido a la pandemia Covid-19 el gobierno}

de México tomó la determinación de interrumpir

las clases presenciales y establecer un sistema

de educación a distancia electrónica y digital,

para la recuperación de contenidos

de aprendizaje (SEP, 2020). el siguiente nivel educativo, además de atender las dificultades que diariamente se presentan respecto a dificultades de conexión, uso de las plataformas educativas y falta de comprensión de contenidos.

La unidad de aprendizaje es Asesoramiento y Orientación Educativa y el campo disciplinar es Orientación Educativa. Las competencias genéricas son: Se autodetermina y cuida de sí. Se conoce y valora a sí mismo y aborda problemas y retos teniendo en cuenta los objetivos que persigue. Los atributos son: Enfrenta las dificultades que se le presentan y es consciente de sus valores, fortalezas y debilidades. La competencia disciplinar es: Administra los recursos disponibles teniendo en cuenta las restricciones para el logro de sus metas.

Los contenidos conceptuales, procedimentales y actitudinales son los siguientes:

- Conceptuales. El asesoramiento en manejo de plataformas educativas, aplicaciones de mensajería y recursos de aprendizaje en línea, orientación en el uso de dichas herramientas y la interacción con alumnos, profesores, directivos y padres de familia.

- Procedimentales. El acceso a plataformas educativas, revisión y descarga de contenidos, comportamiento en clases virtuales, aprovechamiento de recursos educativos en línea.

- Actitudinales. La comunicación asertiva, motivación, empatía, resiliencia, solidaridad.

La educación a distancia es una alternativa para las personas que no pueden trasladarse a las escuelas y 
que requieren de educación, esto para alfabetizarse y cursar la educación básica en busca de mejores oportunidades de desarrollo, para ampliar sus conocimientos y buscar un mejor empleo o simplemente para conocer de temas que le son ajenos o en los cuales desea especializarse.

Hoy en día, los medios de comunicación masiva brindan una amplia gama de herramientas para acceder al conocimiento y aprender nuevos contenidos de diversa índole, desde tutoriales para cocinar hasta la resolución de problemas matemáticos, es decir, que las posibilidades de aprendizaje están al alcance de quien tenga una conexión a internet y un equipo que le permita conectarse a esta modalidad de estudio.

Ahora, en el caso de buscar la certificación de estos estudios, también hay una gran gama de posibilidades ofertadas por las universidades a través de plataformas propias, en donde se diseña un programa de estudio con base en lecturas, foros y clases virtuales para abstraer conocimientos, someterse a alguna prueba y certificarse.

Esto ha ocurrido en nuestro país desde tiempo atrás, dado que la educación a distancia inició por la necesidad de alfabetizar poblaciones rurales. En 1941 se creó la Escuela de Radio de Difusión Primaria para Adultos, en 1944 se fundó el Instituto Federal de Capacitación del Magisterio con cursos por correspondencia a maestros que capacitaron campesinos, y en 1966 inició, mediante una fase de prueba, la Telesecundaria con 83 estudiantes mayores de 12 años a cargo de telemaestros y un profesor-monitor por grupo (Navarrete y Manzanilla, 2017).

\section{Las escuelas públicas representan}

\section{alrededor de 90\% de la matrícula de educación}

primaria y secundaria, $85 \%$ de preescolar

y $80 \%$ de educación media superior y la asistencia a la educación primaria es prácticamente universal (INEE, 2019).

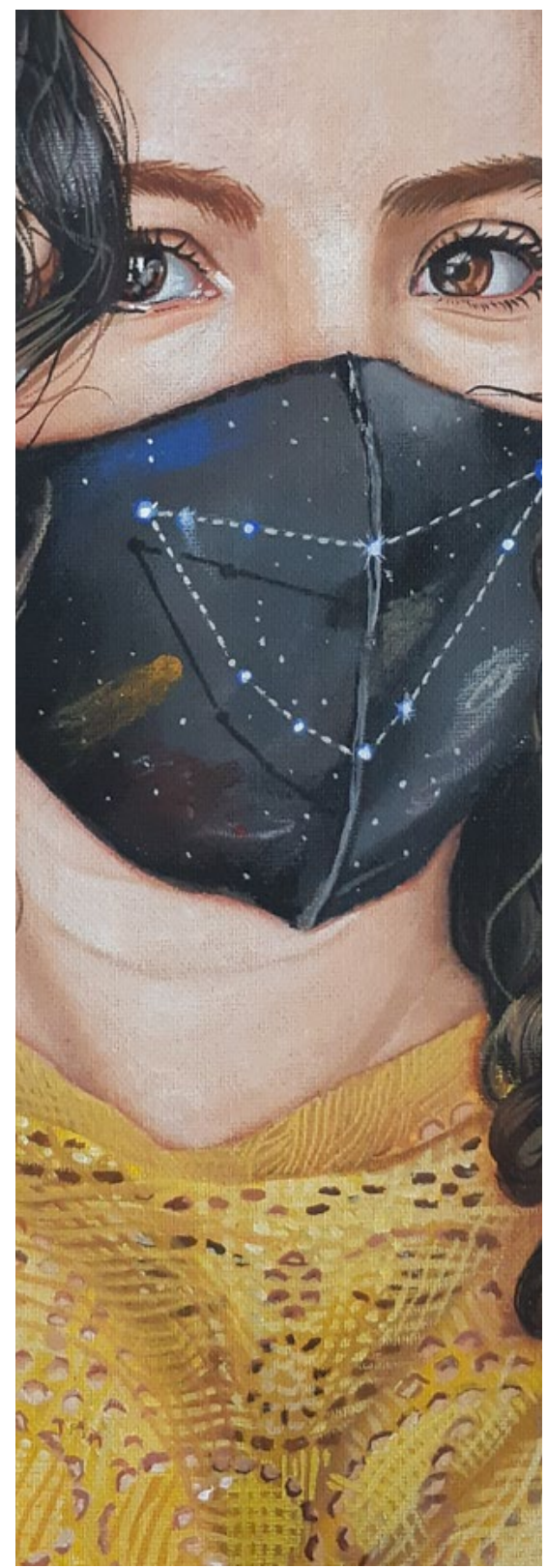

De esta manera entendemos la educación a distancia como una solución a los problemas de cobertura a un número elevado de personas, quienes desean beneficiarse de los avances pedagógicos, científicos y técnicos, pero que les son inaccesibles por su ubicación geográfica o los elevados costos que implica un desplazamiento frecuente 0 definitivo a las escuelas (Ministerio de Educación Nacional, 2009. En: Yong, Nagles, Mejía, Chaparro, 2017).

Considerando que según datos del informe La Educación Obligatoria en México, Informe 2019, las escuelas públicas representan alrededor de $90 \%$ de la matrícula de educación primaria y secundaria, $85 \%$ de preescolar y $80 \%$ de educación media superior y que la asistencia a la educación primaria es prácticamente universal (INEE, 2019), la educación a distancia se ha destinado a cubrir necesidades de aprendizaje en su mayoría de adultos, recién egresados del bachillerato, personas que por diversas circunstancias no cursaron estudios en su juventud o profesionistas en busca de especialización académica. Hoy en día se ven favorecidos por la educación 


\section{En México identifican como principales}

\section{problemas al conectarse a la red la lentitud}

en la transferencia de la información (50.1\%),

interrupciones en el servicio (38.6\%) y exceso

de información no deseada (25.5\%) (INEGI, 2020).

virtual o en línea, que busca propiciar espacios de formación, apoyándose en las TIC para instaurar una nueva forma de enseñar y de aprender (Ministerio de Educación Nacional, 2009. En: Yong, Nagles, Mejía, Chaparro, 2017), las universidades son quienes llevan la pauta en estos servicios educativos que brindan la posibilidad de estudiar a esta población.

Derivado de la pandemia Covid-19, que trasladó a toda la población a la educación a distancia, considerando como primera opción la educación virtual y apoyándose en medios de comunicación como el radio y la televisión, surge la necesidad de que se construya una estrategia nacional que cubra la falta de experiencia en el uso de este tipo de tecnologías y en esta modalidad de trabajo escolar. Las dificultades de cobertura y conexión en nuestro país son enormes, dado que los usuarios de internet en México identifican como principales problemas al conectarse a la red, la lentitud en la transferencia de la información (50.1\%), interrupciones en el servicio (38.6\%) y exceso de información no deseada (25.5 por ciento) (INEGI, 2020).
Ante este panorama, es que cada centro escolar debe adecuarse a la estrategia nacional, pero desarrollando sus propias formas de enfrentar esta situación, buscando alternativas para brindar la atención educativa según su propio contexto y la nueva realidad que viven estudiantes y docentes, a partir de las desigualdades que evidencia y potencia la pandemia Covid-19.

Esto con la intención de que las ventajas de la educación a distancia puedan trasladarse a esta estrategia emergente de atención educativa, tales como apertura para atender estudiantes a

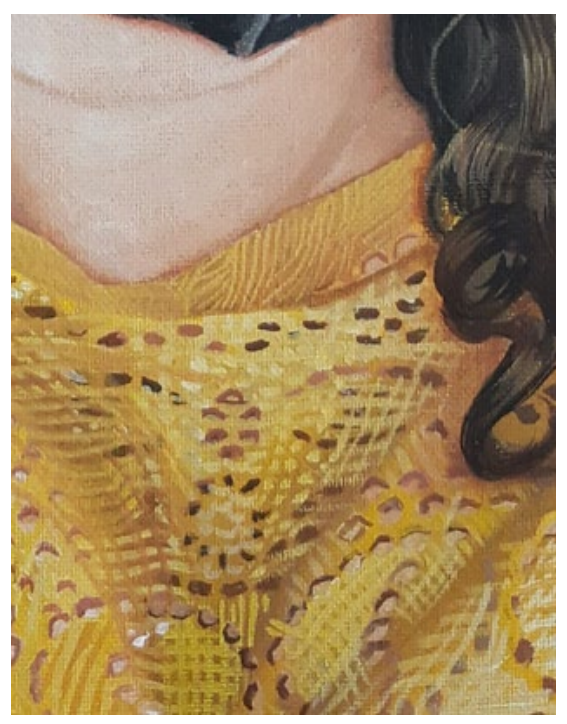

los cuales no se les exige estar presentes en un solo lugar, flexibilidad para que puedan seguir sus estudios sin los rígidos requisitos de espacio, asistencia, tiempo y ritmo de trabajo, la eficacia de convertir al alumno en el centro del proceso de aprendizaje, el autocontrol al fortalecer la capacidad de autogestión del tiempo, del esfuerzo personal y de la conformación de itinerarios formativos (García, 2017).

El objetivo es que los estudiantes desarrollen habilidades y actitudes necesarias para cursar sus clases a distancia y manejar de manera adecuada las plataformas de aprendizaje, así como responder de manera resiliente ante las dificultades que emanan de esta modalidad de aprendizaje, aunado a los efectos familiares, personales, económicos y laborales de la pandemia Covid-19.

\section{METODOLOGÍA}

Dada la emergencia sanitaria, esta estrategia se fue desarrollando conforme empezó el confinamiento, es decir, profesores y alumnos fuimos aprendiendo a trabajar de esta manera con el paso de los días, lo cual generó diversas dificultades académicas, perso-

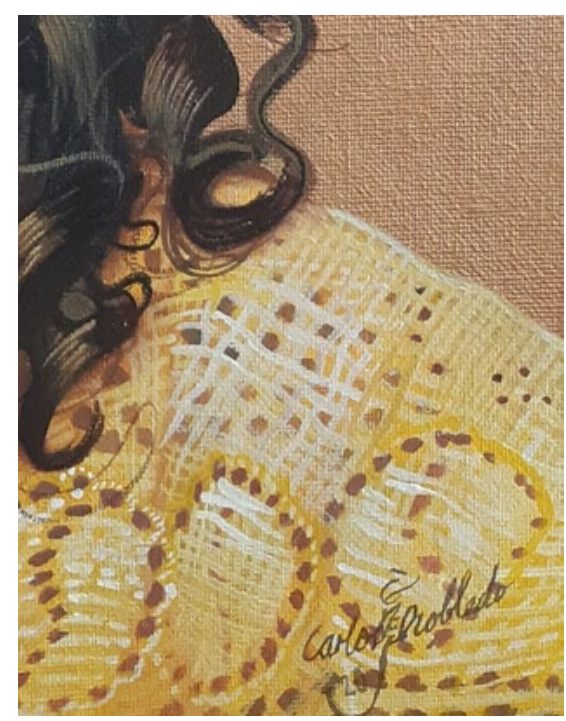




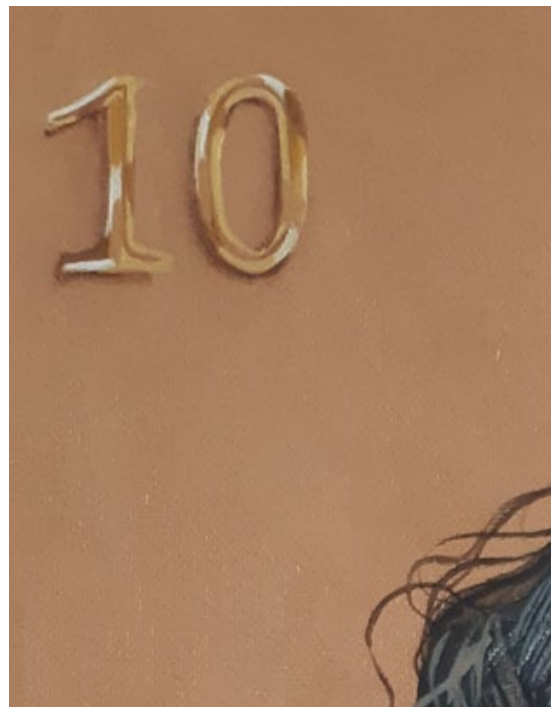

nales, de conexión, de uso de plataformas y de comunicación que debían ser atendidas en su conjunto.

Debido a esto, las distintas áreas escolares han cumplido una función estratégica en esta situación, en el caso de la Orientación Educativa, se convirtió en el puente institucional entre los estudiantes y la escuela, brindando atención y solución a las diversas problemáticas de manera colaborativa con directivos y docentes.

Se aplicó un cuestionario de diagnóstico al inicio del presente ciclo escolar, en donde se recopiló la información de aspectos socioeconómicos y de aprendizaje ocurridos durante el confinamiento debido a la pandemia Covid-19.

La estrategia de atención a distancia con motivo del confinamiento ante la pandemia Covid-19 se está llevando a cabo en dos grupos de segundo grado de la Escuela Preparatoria Oficial No. 257. Los grupos están compuestos por 104 alumnos, de los cuales 49 son hombres y 55 mujeres, con una edad entre 15 y 18 años. La estrategia metodológica de atención que fue

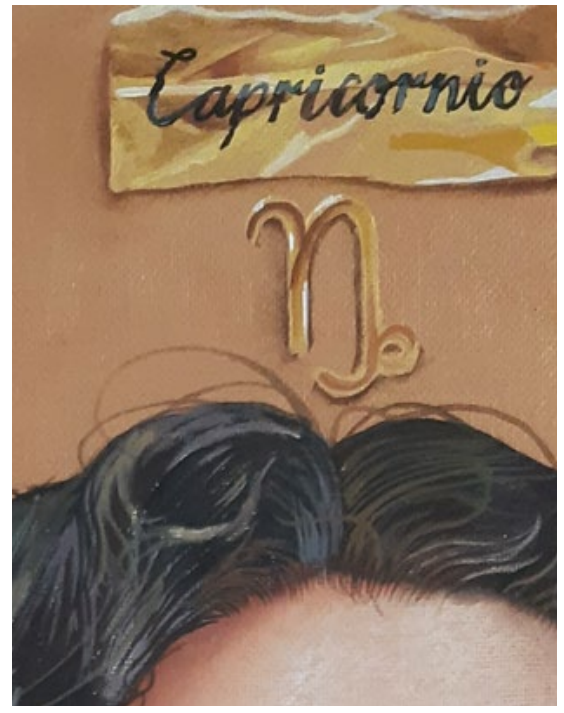

diseñada contempla los siguientes 6 aspectos:

1. Introducción al uso de plataformas.

2. Comunicación con el cuerpo colegiado.

3. Atención a dificultades técnicas.

4. Acompañamiento al proceso de aprendizaje.

5. Seguimiento a procesos administrativos.

6. Vinculación con padres de familia.

\section{INTRODUCCIÓN}

\section{AL USO DE PLATAFORMAS}

El uso de la plataforma classroom era utilizado por algunos docentes de la institución como complemento para las actividades presenciales, servía para subir algunos archivos y consultar algunos vídeos, su uso era esporádico, a partir del confinamiento, no hubo posibilidad de capacitar a estudiantes y docentes en su uso, de manera que por medio de tutoriales todos fuimos aprendiendo.

En el caso de los estudiantes, nos hicieron llegar cuentas institucionales por parte del gobierno del Estado de México para acceder a classroom, el Departamento de Orientación Educativa del plantel del cual formo parte, procedió a enviar dichas cuentas a los estudiantes y tutoriales para su uso, paulatinamente comenzaron a usarlas, entraron a sus aulas y comenzaron con las actividades a distancia, parte de nuestra labor fue informar a los estudiantes cómo utilizar la plataforma para tomar sus clases y atender las dudas que surgieran por la falta de experiencia en su uso.

\section{COMUNICACIÓN}

\section{CON EL CUERPO COLEGIADO}

Se informa a docentes las dificultades que experimentan los alumnos en el trabajo a distancia, se llegan a 


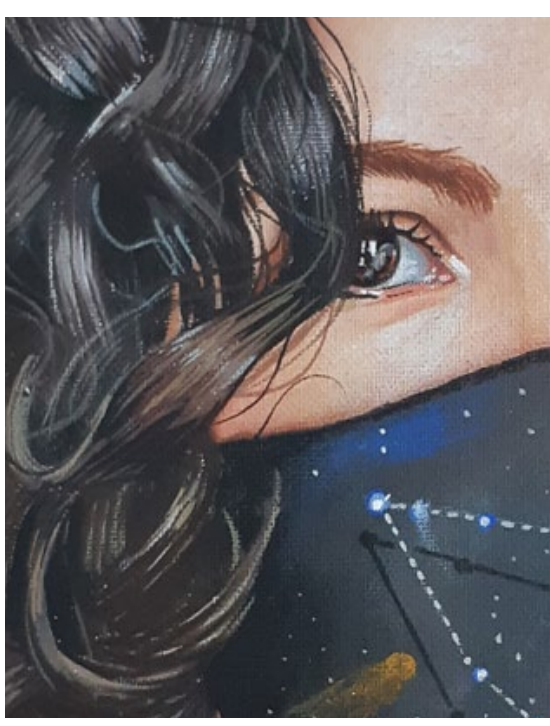

acuerdos en la atención a estos casos y se comunican dichos acuerdos a los estudiantes y padres de familia, se apoya a los docentes al informar a los estudiantes de indicaciones específicas para determinadas actividades, tal es el caso de las clases virtuales y las condiciones de su uso, los horarios de las mismas y se envían los enlaces para acceder a ellas, además se informa de los fallos en las conexiones y las dificultades que reportan los estudiantes para acceder a dichas clases tales como falta de luz, de internet, problemas familiares, traslado a otros Estados de la República, entre otros

Se brinda apoyo en el abordaje de casos específicos en donde se observa ausencia de actividades escolares y se da seguimiento a esos casos con el apoyo del padre de familia para procurar que nadie se quede rezagado.

\section{ATENCIÓN A DIFICULTADES TÉCNICAS}

Dadas las dificultades de conexión 0 falta de la misma, problemas con los equipos de cómputo o teléfonos celulares, que las cuentas se bloquean y no permitan el acceso a plataforma, se recauda esta información y se notifica

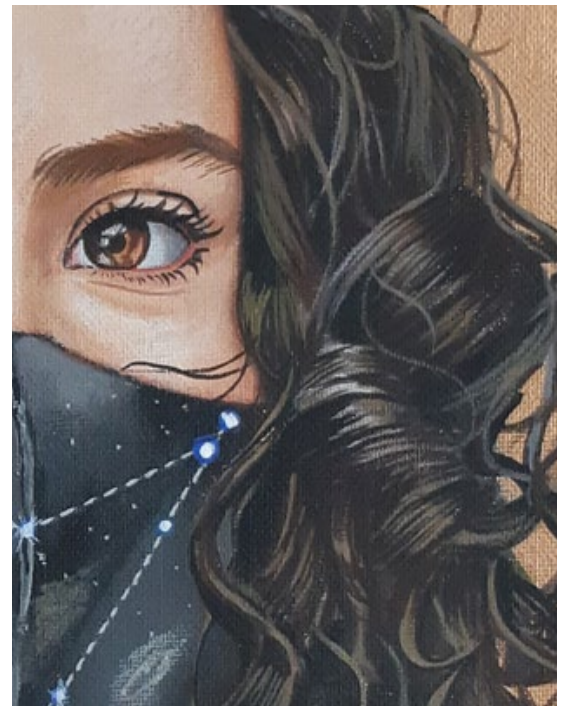

a los directivos del plantel, quienes a su vez establecen comunicación con el departamento encargado de dar soporte a la estrategia de educación a distancia y se solicitan las correcciones. Una vez entregadas, se hacen llegar a los estudiantes y se les indica de qué manera acceder y las precauciones que deben tomar para evitar en la medida de lo posible este tipo de situaciones. Es una actividad recurrente y que requiere de atención prioritaria.

\section{ACOMPAÑAMIENTO}

\section{AL PROCESO DE APRENDIZAJE}

Se brinda atención a dudas en los contenidos de las asignaturas, se informa a los docentes de dichas situaciones y se llegan a acuerdos para atender este tipo de dificultades, se solicita el apoyo de directivos para atender las dificultades que los estudiantes reportan en su proceso de aprendizaje y se mantienen abiertos los canales de comunicación para abordar este tipo de dificultades a la brevedad posible.

\section{SEGUIMIENTO}

\section{A PROCESOS ADMINISTRATIVOS}

En esta parte, se atienden solicitudes de constancias de estudios para diversos trámites las cuales a su vez se so- licitan al personal directivo, se llevan a cabo los procesos de inscripciones y las dificultades que se derivan en la entrega de documentos por diversas causas, se notifica a los directivos de la presencia de alumnos en la plataforma y en clases virtuales, se da seguimiento y apoyo al proceso de becas.

\section{VINCULACIÓN}

\section{CON PADRES DE FAMILIA}

Se establece comunicación con el padre de familia para mantenerlo informado de las situaciones que se van dando en las clases a distancia, el proceso de becas, las inscripciones y se atiende de manera personal casos como dificultades en el acceso a la plataforma, entrega de actividades, consulta de calificaciones y situaciones personales y familiares que impactan en el desempeño de los estudiantes (alumnos trabajando, pérdida de empleo, contagios de Covid-19 en la familia, ansiedad por la carga de trabajo escolar, entre otras).

\section{CONOCIMIENTOS PREVIOS}

El uso de la plataforma classroom, el uso de aplicaciones de mensajería como Gmail y WhatsApp, el envío de actividades escolares a través de estos medios y el uso del teléfono celular como medio de interacción.

\section{AMBIENTE DE APRENDIZAJE}

Tolerante, solidario y empático, comprendiendo las distintas realidades que convergen en un ambiente intercultural como lo es la escuela, además de las circunstancias socioeconómicas diversas que se viven en los distintos hogares y las posibilidades que limitan estudiar a distancia. Se llevó a cabo la estrategia de atención en los 6 aspectos de la propuesta metodológica: Introducción al uso de plata- 


\section{El rendimiento de los estudiantes en su percepción}

\section{de logro, el 55.3\% considera que logró aprender}

pero tienen algunas dudas en ciertos temas

y el 7.4\% aprendió de forma adecuada.

formas; Comunicación con el cuerpo colegiado; Atención a dificultades técnicas; Acompañamiento al proceso de aprendizaje; Seguimiento a procesos administrativos; Vinculación con padres de familia a través de comunicados y atención personalizada en Gmail y WhatsApp, además de reuniones virtuales a través de Meet.

\section{RESULTADOS Y CONCLUSIONES}

Los resultados de la estrategia de intervención, se aplicó mediante un cuestionario diagnóstico al inicio del presente ciclo escolar, en donde se recopila información de aspectos socioeconómicos y de aprendizaje ocurridos durante el confinamiento debido a la pandemia Covid-19, se observó que el $45.7 \%$ de los alumnos considera que su desempeño académico fue bueno, el $38.3 \%$ regular, $6.4 \%$ excelente, $8.5 \%$ nada bueno y $1.1 \%$ malo, es decir, que a pesar de las circunstancias de educación a distancia y las dificultades experimentadas, la gran mayoría considera desempeños académicos de regulares a buenos.

El 55.3\% indica que entregó todas 0 la mayoría de las actividades en los tiempos establecidos, el $29.8 \%$ indi- can haber entregado algunas actividades en estos lapsos de tiempo, el 8.5\% reporta entregar a destiempo y sólo el $6.4 \%$ que pasaban lapsos de tiempo sin entregar actividades, esto implica que en general hubo buen ritmo de trabajo, situación observada en el rendimiento de los estudiantes y en su percepción de logro, dado que el $55.3 \%$ considera que logró aprender pero tienen algunas dudas en ciertos temas y el $7.4 \%$ aprendió de forma adecuada.

Aun así es importante considerar las dificultades socioeconómicas que derivan de esta pandemia, debido a que el $31.9 \%$ indica que no aprendió del todo bien y que requiere un mayor esfuerzo junto al $5.3 \%$ que reportó estar ausente de sus clases, situaciones que se relacionan con el hecho de que el $16.3 \%$ tuvo que salir a trabajar para aportar al gasto familiar, el 12.8\% reporta que la persona que se hace cargo económicamente de ellos trabaja de manera presencial 0 a distancia pero le redujeron el salario, el $2.1 \%$ fue descansado y está en espera de ser llamado a laborar, y el $4.3 \%$ fue despedido y actualmente no labora. Además de que el $46.8 \%$ reporta fallas en su conexión a internet, el $10.6 \%$ reporta fallas en el servicio de energía eléctrica y el $10.6 \%$ fallas en su celular, el principal medio para conectarse a las actividades a distancia (80.9\%).

Estos parámetros reflejan la realidad de nuestra sociedad mexicana, que en algunas zonas y en ciertos aspectos las desigualdades se hacen más presentes ante la pandemia Covid-19, y que no puede mirarse de la misma manera lo que ocurre en las grandes ciudades 0 en zonas rurales, tampoco al interior de una misma ciudad, dado que Ecatepec es parte de la zona metropolitana de la capital del país, y aun así existen este tipo de disparidades en las realidades que viven uno y otros.

Las estrategias de intervención deben ser conscientes de estas realidades y adecuarse a ellas, así como fomentar la empatía, solidaridad y compromiso educativo para que todos los estudiantes puedan participar en las actividades a distancia y desarrollarse de la mejor manera posible.

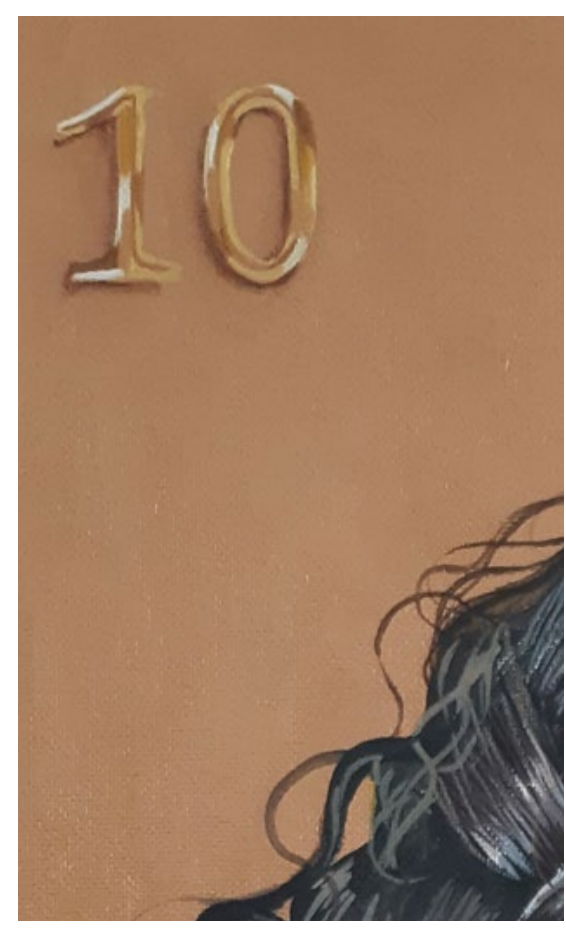




\section{REFERENCIAS}

García, L. (2017). Educación a distancia y virtual: calidad, disrupción, aprendizajes adaptativo y móvil. RIED. Revista Iberoamericana de Educación a Distancia, 20 (2), 9-25. Disponible en: https://www.redalyc.org/articulo. oa? id $=3314 / 331453132001$

INEE (2019). La Educación Obligatoria en México, Informe 2019. Disponible en: https://www.inee.edu.mx/medios/informe2019/stage 02/index.html

INEGI (2020). En México hay 80.6 millones de usuarios de internet y 86.5 millones de usuarios de teléfonos celulares. ENDUTIH 2019. Comunicado de prensa núm. 103/20 del 17 de febrero de 2020. Disponible en: https://www.inegi.org. $\mathrm{mx} /$ contenidos/saladeprensa/boletines/2020/OtrTemEcon/ENDUTIH_2019.pdf

Navarrete, Z. y Manzanilla, H. M. (2017). Panorama de la Educación a Distancia en México. Revista Latinoamericana de Estudios Educativos(Colombia), 13 (1),6582.Disponible en: https://www.redalyc.org/articulo.oa?id=1341/134152136004 SEP (2020). Comunicado conjunto No. 3 Presentan Salud y SEP medidas de prevención para el sector educativo nacional por COVID-19. Disponible en: https://www.gob.mx/sep/es/articulos/comunicado-conjunto-no-3-presentan-salud-y-sep-medidas-de-prevencion-para-el-sector-educativo-nacional-por-covid-19?idiom=es

Yong, E., Nagles, N., Mejía, C. y Chaparro, C. E. (2017). Evolución de la educación superior a distancia: desafíos y oportunidades para su gestión. Revista Virtual Universidad Católica del Norte, (50), 81-105. Disponible en: https://www. redalyc.org/articulo.0a?id=1942/194250865006

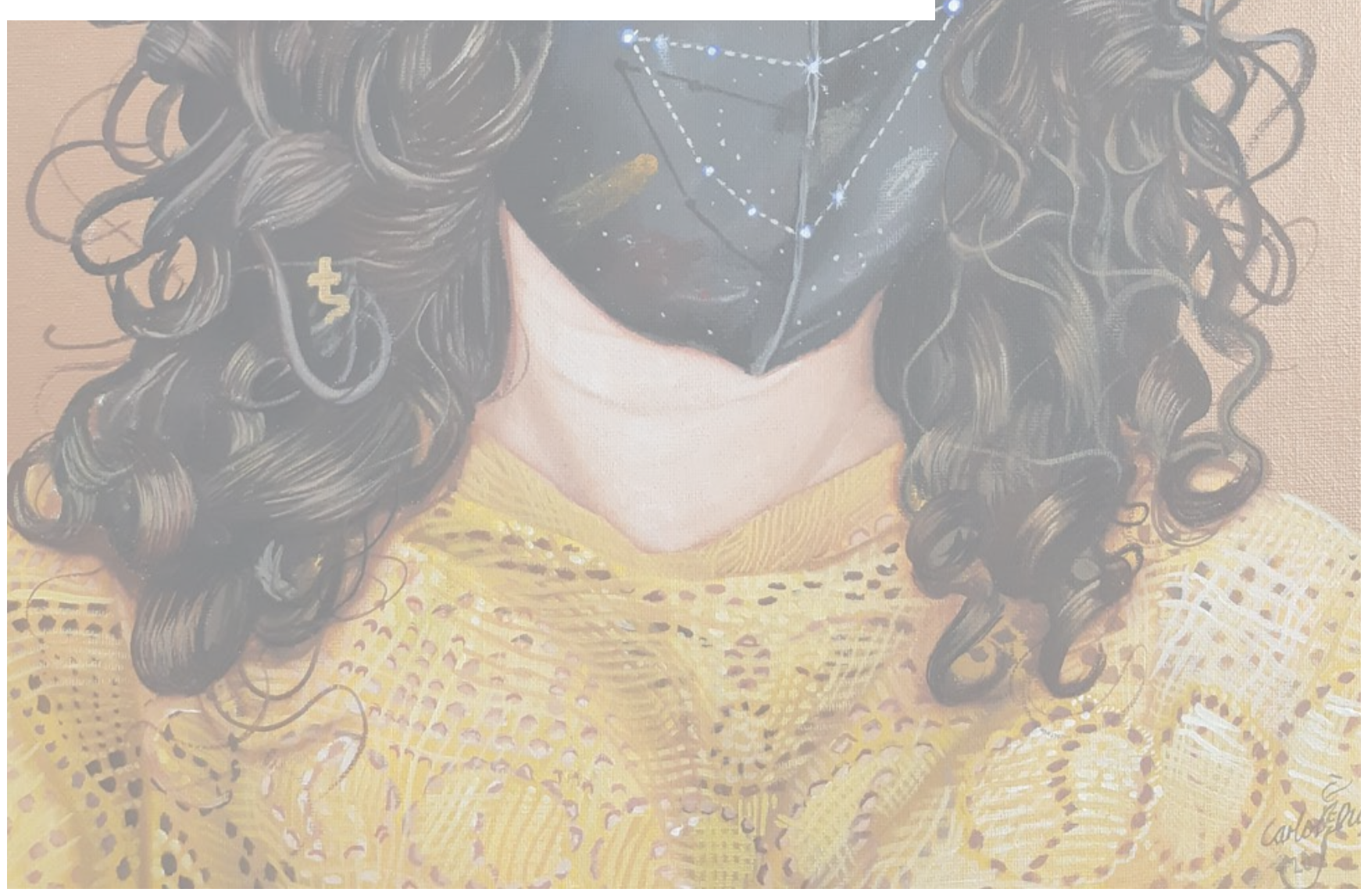




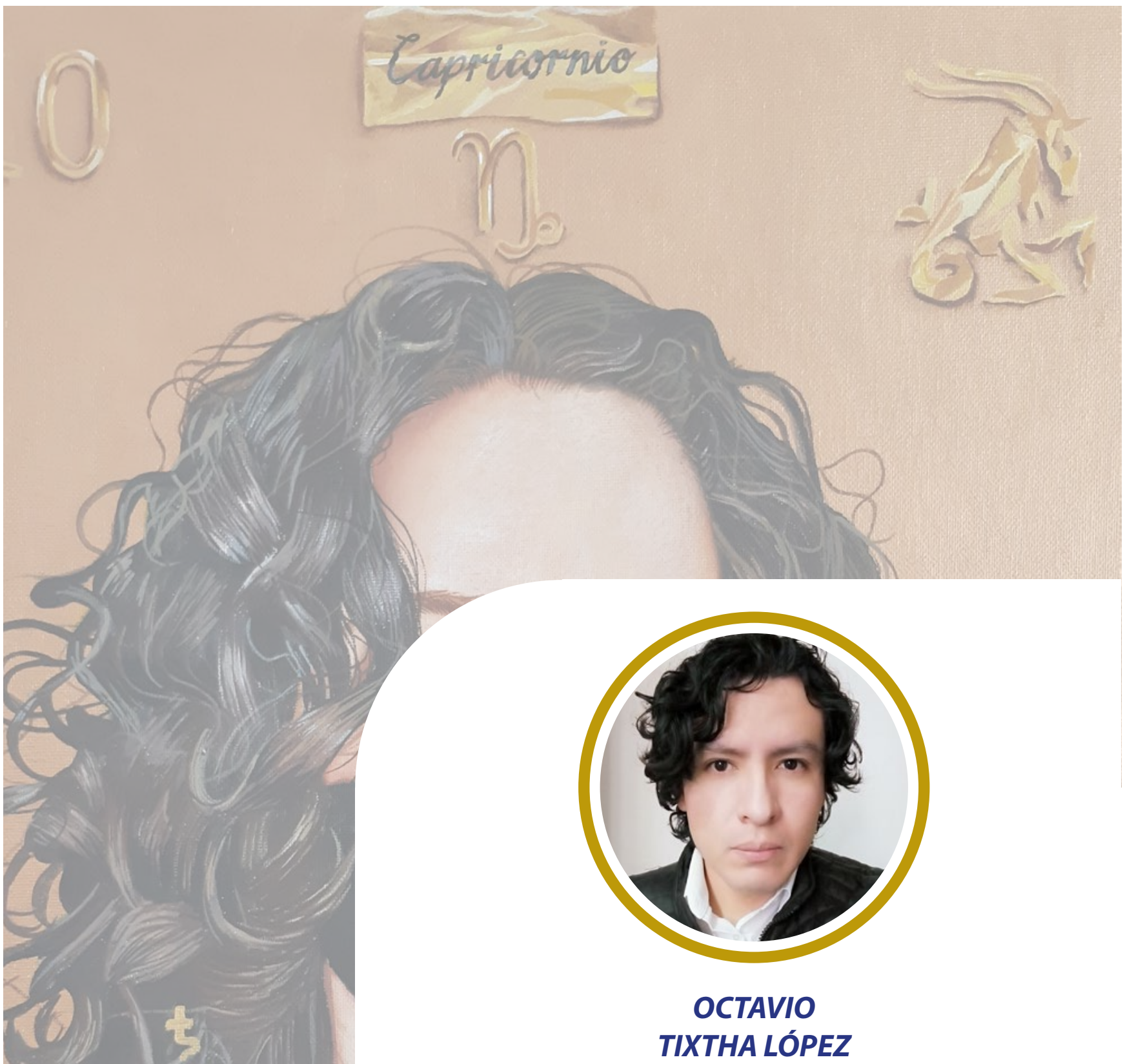

Es Licenciado en Psicología (UNAM), Maestro en Gestión de la Convivencia en la Escuela. Violencia, Derechos Humanos y Cultura de Paz (UPN) y Maestro en Educación (UVM). Diplomado en Acoso escolar y consumo de drogas y Educación para los Derechos Humanos. Orientador educativo de bachillerato en el Estado de México, ponente y tallerista en congresos educativos con publicaciones de artículos de investigación en México y España.

Correo Electrónico: nincrepusculo@hotmail.com

Fecha de Recepción: Octubre 2020 Fecha de Aprobación: Diciembre 2020 


\section{$(36)$ \\ Carlos Enrique Robledo Moreno 1981, Monterrey, Nuevo León}

Artista egresado de la Facultad de Artes Visuales de la Universidad Autónoma de Nuevo León. La mayor parte de su obra la realiza en murales por las principales avenidas del municipio de San Nicolás de los Garza, Nuevo León, México. Coordina el programa de Arte Urbano en el Instituto Municipal para el Desarrollo Cultural (IMDEC) de San Nicolás de los Garza. Además, realiza su obra en lienzo y en el arte Madonnari (gis/pavimento). Ha expuesto y pintado de manera local, nacional e internacionalmente en paises como Francia, Italia y Estados Unidos. Ha recibido importantes reconocimientos como en Nocera Superiore, Italia, 2013 (2do. lugar del público) y 2015 (1er lugar del público); y en Wilhelmshaven, Alemania, 2019 (3er Lugar Categoría Libre).

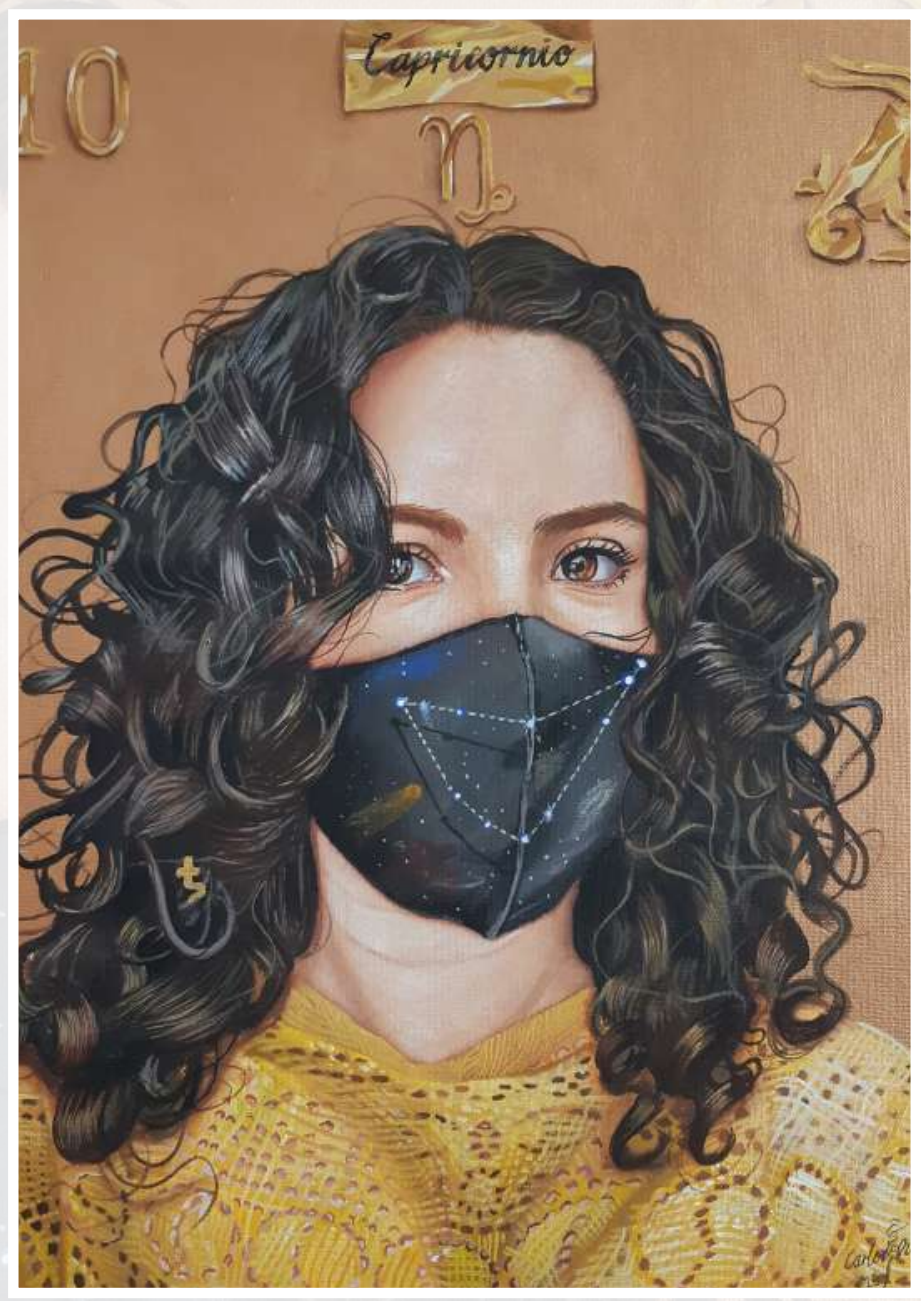

Como artista visual plasmo el retrato y figura humana realista, en conjunto con una simbología acorde a la temática de la obra. Me gusta ver mi obra como todo un proceso, el cual va desde la idea, el boceto, la elección del o la modelo, la toma de una fotografía o bien un dibujo, hasta la realización de la pintura. Generalmente utilizo el acrílico por la rapidez con la que puedo realizar obras en tonos vivos y cálidos. Pienso que en el discurso de la obra que realizo, trato hacer conciencia de lo caótico de la ciudad, del ser humano en sí y de la interacción del individuo con la naturaleza. 